\section{Automated vitrector-assisted iridectomy and phacoemulsification in eyes with coexisting cataract and adherent leucomas}

T Agarwal, V Jhanji, P Dutta and JS Titiyal

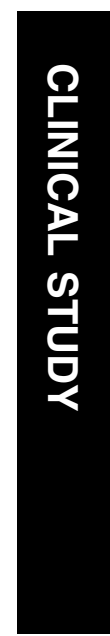

the preferred method for treating cases with corneal scars and coexistent cataract. ${ }^{1-3}$ The scarcity of donor corneal tissue and the risk of graft failure, particularly in high-risk keratoplasty, create the need for alternative techniques for restoring ambulatory vision in such cases. Moreover, the outcome of penetrating keratoplasty is not promising in cases with corneal scarring and adherent leucomas' postcorneal ulcers. ${ }^{4}$ We describe a new technique of automated vitrectorassisted optical iridectomy combined with phacoemulsification and IOL implantation in patients with cataract and coexisting partial corneal opacificaion.

\section{Methods}

A prospective study was conducted at the Rajendra Prasad Centre for Ophthalmic Sciences. Seven eyes of seven patients with adherent leucomas secondary to perforated corneal ulcers and age-related cataracts were enroled for the study. All cases selected for the study had central or paracentral corneal opacities with at least one quadrant of clear peripheral cornea (Figure 1). Informed consent was obtained from the subjects, and Institutional Review Board approval was obtained. The preoperative evaluation included recording of uncorrected visual acuity (UCVA), best-corrected visual acuity (BCVA), visual acuity after dilatation of pupil, refraction using a stenopaic slit, and a detailed slit-lamp examination. Posterior segment evaluation was performed to rule out any posterior segment pathology. Eyes with inaccurate projection of Ophthalmology; Rajendra Prasad Centre for India Institute of Medical Sciences, New Delhi, India

Correspondence: T Agarwal, Department of Ophthalmology; Rajendra Prasad Centre for Ophthalmic Sciences, All India Institute of Medical Sciences,

New Delhi-110029, India Tel: +91 11 26588500; Fax: +911126588919.

E-mail: drtushar@ gmail.com

Received: 20 April 2008 Accepted in revised form: 24 June 2008 Published online: 14 November 2008

This study was presented in part as a poster at the XXIV Congress of the ESCRS London 2006

Proprietary interests: None Research funding: None
Department of Ophthalmic Sciences, All 


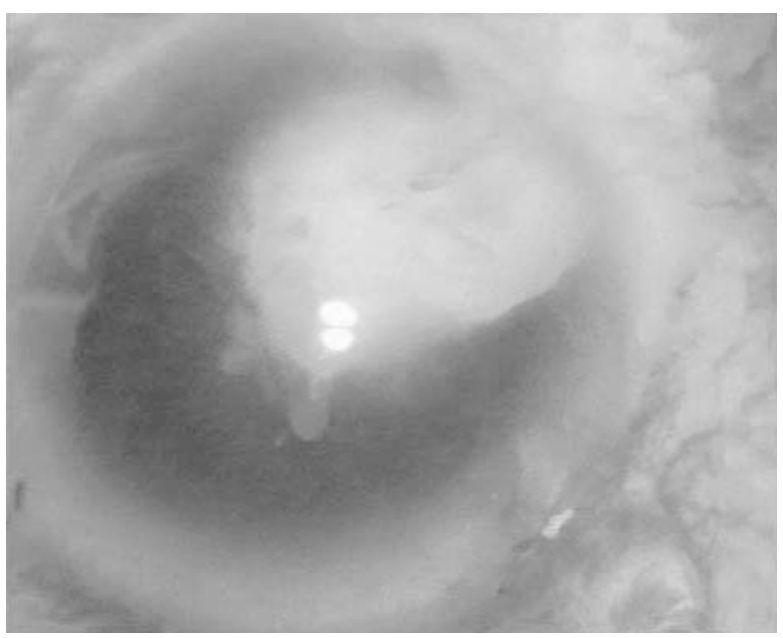

Figure 1 Intraoperative photograph showing corneal opacity with central adherent leucoma and cataract.

light and those with posterior segment pathology were excluded from the study.

\section{Surgical technique}

Intravenous mannitol ( $1 \mathrm{gm} / \mathrm{kg}$ body weight) was given to all the patients $30 \mathrm{~min}$ before the surgery to prevent peroperative intraocular pressure surge. All surgeries were performed under peribulbar anaesthesia using 4-ml lidocaine hydrochloride (xylocaine 2\%, Astra Zeneca, India) and $2 \mathrm{ml}$ of bupivacaine hydrochloride $0.5 \%$ (Sensorcaine, Astra Zeneca, India). A 2.75-mm incision was made on the limbus radially opposite to the site of adherent leucoma using a keratome (Alcon Labs, Fortworth, TX, USA). A $1.2 \mathrm{~mm}$ side port was made about 2-3 h away on the clear cornea. Hydroxypropyl methycellulose solution of 2\% (Visilon, Shah and Shah, India) was injected in the space below the iris to lift it away from the crystalline lens towards the cornea. A 20-gauge aspiration-cutter vitrectomy probe (DORC International, the Netherlands) was inserted into the anterior chamber through the main port. Aspiration $(100 \mathrm{mmHg})$ as well as cutting (700 c.p.m.) was used to release the adherent leucoma while maintaining the anterior chamber depth with the help of continuous infusion from the side port. The aspiration port was occluded by the iris stroma, and the iris was cut using the vitrectomy probe under direct visualization (Figure 2a). The probe was removed and the anterior chamber was irrigated with the help of balanced salt solution (BSS; Alcon, Fortworth, TX, USA) to remove the viscoelastic. Trypan blue $0.01 \%$ (Vision blue; DORC International BV, ZUIDLAND, the Netherlands) was used to stain the anterior capsule. The anterior chamber was filled with
Healon GV (AMO Inc., CA, USA). A continuous curvilinear capsulorrhexis (CCC) was initiated in all the cases using a bent $26-\mathrm{G}$ needle. After partially completing the capsulorrhexis up to one edge of corneal opacity, the margin of capsulorrhexis flap was held firmly with a pair of Uttrata forceps and CCC was continued under the corneal opacity by taking care not to lose hold of the capsulorrhexis flap. The edge of CCC flap was repeatedly grasped and capsulorrhexis was completed. This was followed by hydrodissection and hydrodelineation to achieve free rotation of the nucleus in the capsular bag. Phacoemulsification was performed using primary chop technique and using the Storz protégé machine (Storz Protégé, Bausch \& Lomb, NY, USA; Figure 2b). All manoeuvers during phacoemulsification were performed in the clear window created at the start of the surgery using the automated vitrector. After completing phacoemulsification, bimanual irrigation and aspiration was performed for removal of cortical matter (Figure 2c). The capsular bag was filled with Healon GV (AMO Inc., CA, USA) and a single piece acrylic foldable IOL (ACRYSOF ${ }^{\circledR}$ SA60AT; Alcon laboratories, Fort Worth, TX, USA) was implanted. The viscoelastic was completely removed with irrigation and aspiration. At the end of the surgery, the $2.75 \mathrm{~mm}$ wound as well as the side ports were hydrated with BSS (Figure 2d). An air bubble was left inside the anterior chamber.

Postoperatively, all patients received prednisolone acetate $1 \%$ eye drops (Predacetate, Allergan, India) every $4 \mathrm{~h}$ and moxifloxacin hydrochloride $0.5 \%$ eye drops (Vigamox, Alcon, India) three times a day. Timolol maleate $0.5 \%$ eye drops (Iotim, FDC, India) were prescribed twice a day whenever required. Patients were evaluated on days 1 and 7 , and 6 weeks following surgery.

\section{Results}

The release of iris adherence along with creation of optical iridectomy at the beginning of surgery improved visualization of intraocular structures during cataract surgery (Table 1). Phacoemulsification and IOL implantation could be performed successfully in all seven eyes (Figure 3). No intraoperative or postoperative complications were seen in any patient. The median BCVA improved from 1/60 (range: (light perception) 6/36) preoperatively to $6 / 18$ (range: $6 / 36-6 / 12$ ) at last follow-up (average: 41 days). Two patients complained of glare in bright light in the postoperative period. It was managed adequately after they were prescribed photochromatic glasses. 

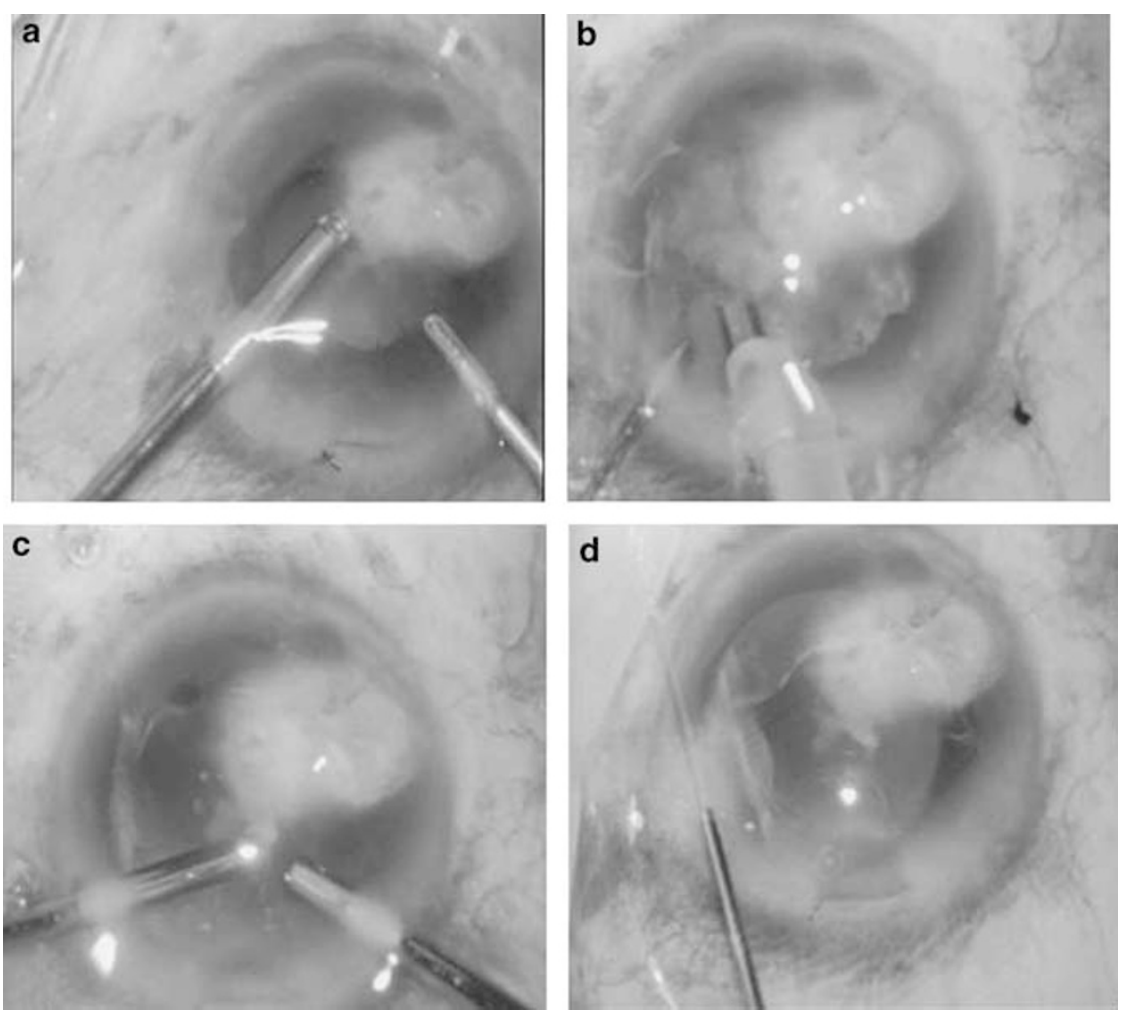

Figure 2 (a) Pupil enlarged with the automated vitrector to create clear area for performing phacoemulsification (intraoperative photograph). (b) Phacoemulsification being performed in the clear area created previously (intraoperative photograph). (c) Bimanual irrigation aspiration for the removal of cortical lens matter (intraoperative photograph). (d) Foldable intraocular lens implanted in the capsular bag (intraoperative photograph).

Table 1 Summary of patients undergoing automated vitrectorassisted iridectomy with phacoemulsification and intraocular lens implantation

\begin{tabular}{lclc}
\hline Patient & Age (years)/sex & Preoperative BCVA & Postoperative BCVA \\
\hline 1 & $47 / \mathrm{M}$ & $6 / 60$ & $6 / 18$ \\
2 & $60 / \mathrm{M}$ & $1 / 60$ & $6 / 36$ \\
3 & $70 / \mathrm{M}$ & $6 / 36$ & $6 / 12$ \\
4 & $45 / \mathrm{M}$ & Light perception & $6 / 36$ \\
5 & $85 / \mathrm{M}$ & $6 / 60$ & $6 / 18$ \\
6 & $68 / \mathrm{M}$ & $1 / 60$ & $6 / 18$ \\
7 & $60 / \mathrm{M}$ & Light perception & $6 / 36$ \\
\hline
\end{tabular}

\section{Discussion}

Allograft corneal transplantation combined with extra capsular cataract extraction and IOL implantation is the preferred method for treating cases with adherent leucomas and co-existent cataract. However, the scarcity of donor corneas, the risk of graft failure, secondary glaucoma, and suture-related problems after a triple procedure necessitate the development of alternative surgical techniques for cases with partial corneal opacification and cataract. Patients with nebulomacular

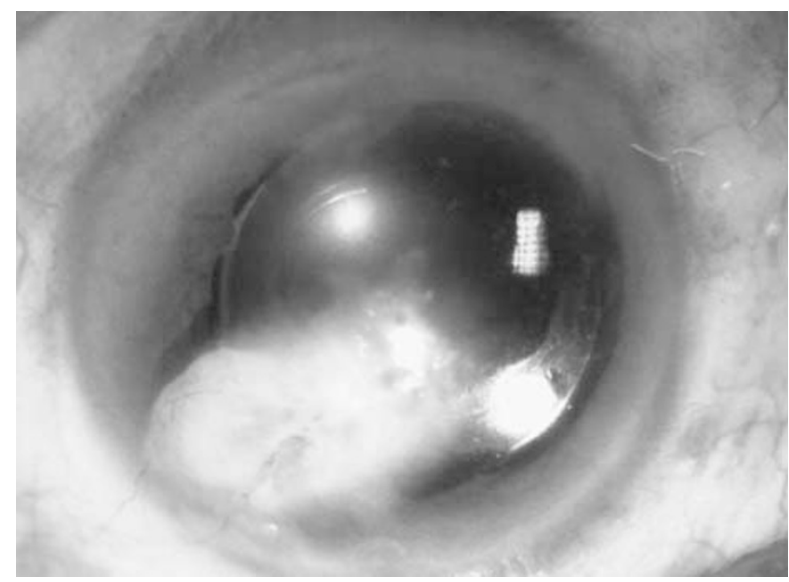

Figure 3 Postoperative clinical photograph showing optical window created after automated vitrector-assisted optical iridectomy and intraocular lens in place (6 weeks).

corneal opacities and visually debilitating cataract may become regain ambulatory vision with cataract surgery alone. ${ }^{5,6}$ However, a successful phacoemulsification may be difficult in cases of leucomatous coneal opacity with adherent leucoma because of poor visualization of the 
is a feasible alternative to a conventional triple procedure in patients with partial central or paracentral corneal opacification and coexisting age-related cataract.

\section{Acknowledgements}

We would like to acknowledge Dr Namrata Sharma for her contribution to the study.

\section{References}

1 Skorpik C, Menapace R, Gnad HD, Grasl M. The triple procedure - results in cataract patients with corneal opacity. Ophthalmologica 1988; 196: 1-6.

2 Arentsen JJ, Laibson PR. Penetrating keratoplasty and cataract extraction: combined vs nonsimultaneous surgery. Arch Ophthalmol 1978; 96: 75-76.

3 Shimmura S, Ohashi Y, Shiroma H, Shimazaki J, Tsubota K. Corneal opacity and cataract: triple procedure versus secondary approach. Cornea 2003; 22: 234-238.

4 Garg P, Krishna PV, Stratis AK, Gopinathan U. The value of corneal transplantation in reducing blindness. Eye 2005; 19: 1106-1114.

5 Sinha R, Sharma N, Vajpayee RB. Visual outcome of cataract surgery with pupillary sphincterotomy in eyes with coexisting corneal opacity. BMC Med 2004; 2: 10.

6 Pandey SK, Werner L, Escobar-Gomez M, Roig-Melo EA, Apple DJ. Dye-enhanced cataract surgery. Part 1: anterior capsule staining for capsulorrhexis in advanced/white cataract. J Cataract Refract Surg 2000; 26: 1052-1059.

7 Dada VK, Sharma N, Sudan R, Sethi H, Dada T, Pangtey MS. Anterior capsule staining for capsulorrhexis in cases of white cataract: comparative clinical study. J Cataract Refract Surg 2004; 30: 326-333.

8 Titiyal JS, Sinha R, Sharma N, Vajpayee RB. Dye-assisted small incision cataract surgery in eyes with cataract and coexisting corneal opacity. Eye 2006; 20: 386-388.

9 Sinha R, Sharma N, Vajpayee RB, Titiyal JS. Trypan blue-assisted high-volume cataract surgery in a peri-urban eye hospital in India. Trop Doct 2006; 36: 63.

10 Bhartiya P, Sharma N, Ray M, Sinha R, Vajpayee RB. Trypan blue assisted phacoemulsification in corneal opacities. Br J Ophthalmol 2002; 86: 857-859.

11 Melles GRJ, de Waard WT, Pameyer JH, Houdijn Beekhuis W. Trypan blue capsule staining to visualize the capsulorrhexis in cataract surgery. J Cataract Refract Surg 1999; 25: 7-9. 